\title{
1 Neural Correlates of Causal Confounding
}

2 Mimi Liljeholm

3 Department of Cognitive Sciences, University of California, Irvine

4

5 Corresponding author:

Mimi Liljeholm

Department of Cognitive Sciences

$9 \quad$ University of California

Irvine, CA, 92697-5100

11

E-mail: m.liljeholm@uci.edu

12

13 Keywords: Causal induction, Uncertainty, confounding, prediction errors, fMRI 


\section{Abstract}

15 As scientists, we are keenly aware that if putative causes perfectly co-vary, the

16 independent influence of neither can be discerned - a "no confounding" constraint on

17 inference, fundamental to philosophical and statistical perspectives on causation.

18 Intriguingly, a substantial behavioral literature suggests that naïve human reasoners,

19 adults and children, are tacitly sensitive to causal confounding. Here, a combination

20 of $\mathrm{fMRl}$ and cognitive computational modeling was used to investigate neural

21 substrates mediating such sensitivity. While being scanned, participants observed

22 and judged the influences of various putative causes with confounded or non-

23 confounded, deterministic or stochastic, influences. During judgments requiring

24 generalization of causal knowledge from a feedback-based learning context to a

25 transfer probe, activity in the dorsomedial prefrontal cortex (DMPFC) was better

26 accounted for by a Bayesian causal model, sensitive to both confounding and

27 stochasticity, than a purely error-driven algorithm, sensitive only to stochasticity.

28 Implications for the detection and estimation of distinct forms of uncertainty, and for a

29 neural mediation of domain general constraints on causal induction, are discussed. 


\section{Introduction}

34 Consider two scenarios involving a target cause, $C$, an alternative cause, $A$, and the

35 presence $(+)$ or absence $(-)$ of some effect. In the first scenario, the effect occurs

36 when $C$ and $A$ are presented in combination, but not when $A$ is presented alone $[A-$,

$37 A C+]$. In the second scenario, the $A$ - trials are removed, so that both $C$ and $A$ occur

38 only in combination with each other $[A C+]$. How would your judgment about the

39 influence of $C$ differ across these two scenarios? Philosophers, scientists and

40 statisticians alike recognize that perfect co-variation of $C$ and $A$ in the second scenario

41 occludes the independent influences of each putative cause, rendering any judgment

42 about their respective effects fraught with uncertainty. Importantly, naïve human

43 reasoners also appear to, tacitly, apply this domain-general constraint on causal

44 induction, as evidenced by behavioral data showing sensitivity to confounding in a

45 wide range of causal and predictive judgments, by children as well as adults

46 (Spellman, 1996; Kushnir and Gopnik, 2005; Meder et al., 2006; Schulz and Bonawitz,

47 2007; Schulz et al., 2007; Liljeholm, 2015). Very little is known, however, about the

48 neural substrates mediating sensitivity to, and uncertainty associated with, causal

49 confounding. The current study aims to identify such neural computations.

50 A second source of uncertainty in causal and predictive inference, that has been

51 thoroughly investigated both behaviorally and neutrally, is the stochasticity, or

52 variance, of the outcome variable, which is greatest when the probability distribution

53 over possible outcome states is uniform. As with causal confounding, behavioral

54 sensitivity to outcome stochasticity is well established (e.g., Holt \& Laury, 2002).

55 Moreover, several neuroimaging studies have implicated the dorsomedial prefrontal 
56 cortex (DMPFC), anterior insula, thalamus and dorsolateral prefrontal cortex (DLPFC)

57 in the neural representation of outcome stochasticity (Volz et al., 2003; Huettel et al.,

58 2005; Grinband et al., 2006; Abler et al., 2009). An important open question is

59 whether, and which of, these neural regions also implement signals associated with

60 causal confounding. In the current study, participants were scanned with functional

$61 \mathrm{MRI}$ while observing and judging the influences of various putative causes with

62 confounded or non-confounded, deterministic or stochastic, influences.

63 Note that, if a particular set of confounded causes always occur in the same

64 configuration, and the only goal is to predict the state of the outcome based on that 65 particular configuration, then confounding does not pose a problem. Indeed, most

66 individual, non-confounded, causes can presumably themselves be broken into a set

67 of always co-occurring, and thus confounded, elements. The critical question, thus, is

68 whether it is necessary to tease apart the influences of individual elements, such as

69 when a confounded cause suddenly occurs on its own, or in a novel combination with

70 some other cause. In the current study, participants were required both to make

71 predictions about the outcome given a particular, recurring, configuration of

72 confounded causes and, in other instances, to make explicit judgments about the

73 individual influences of those same causes. Only in the latter case would uncertainty

74 due to confounding be warranted.

75 Formally, two dominant approaches to predictive uncertainty can be discerned in the 76 psychological literature. First, in associative learning theory, an error-driven

77 representation of uncertainty about a cue's predictive strength is captured by the 78 "Pearce-Hall" (PH) algorithm, which relates the "associability" of a cue to a weighted 
79 average of the absolute prediction error on previous trials involving that cue. Since

80 the frequency and size of prediction errors increases with unpredictability, this quantity

81 is proportional to the stochasticity of the outcome. Conversely, in Bayesian Causal

82 Models, uncertainty about the predictive strength of a cause is reflected in the entropy

83 of the posterior distribution over its possible strengths, which depends on the variance

84 of the effect variable, but also on the independent occurrence of alternative causes.

85 While both the associative and Bayesian causal model predicts sensitivity to

86 stochasticity, only the causal model accounts for uncertainty due to causal

87 confounding. Here, a combination of neuroimaging and cognitive modeling was used

88 to dissociate neural signals scaling with error-driven and causal uncertainty. In

89 particular, judgments requiring generalization of causal knowledge from a feedback-

90 based learning context to a transfer probe were expected to elicit strong neural

91 responses to both stochasticity and confounding.

\section{Materials \& Methods}

93 Participants. 20 healthy normal volunteers (mean age $=20.9 \pm 2.4$, range: $18-27,12$

94 females) participated in the study. A power analysis performed on data from a pilot 95 study on error-driven uncertainty, described in detail below, indicated that a sample

96 size of 16 would yield a power of 0.9 at a Gaussian random field theory corrected

97 threshold of 0.05 in regions of interest. One participant was excluded prior to any

98 analyses due to excessive head movement $(>6 \mathrm{~mm})$, leaving a sample size of 19 . The

99 volunteers were pre-assessed to exclude those with a history of neurological or 
100 psychiatric illness. All subjects gave informed consent and the study was approved by

101 the Institutional Review Board of the University of California, Irvine.

102 Task \& procedure. Participants were scanned with functional MRI while performing a

103 causal induction task in which they assumed the role of a research scientist assessing

104 the influence of various allergy medicines on headache - a potential side effect. At

105 the beginning of the study, participants were instructed that each medicine could

106 either produce headache or have no influence on headache (i.e., there were no

107 preventive causes) and, further, that the influence of a given medicine might be

108 stochastic, so that even if that medicine was indeed a cause of headache, it may still

109 not produce headache every time it was administered. Three target medicines, C, D

110 and S, mnemonically labeled here to indicate "confounded", "deterministic" and

111 "stochastic" influences respectively, occurred only in combination with some

112 alternative medicine during feedback-based learning. Specifically, with,+- and \pm

113 respectively indicating a $1.0,0$ and 0.5 probability of the effect, and with lower case

114 letters indicating non-target causes, there were 5 different types of medicine

115 treatments: e-, s' \pm , De+, Se \pm and Cc'+. None of the allergy patients had headache in

116 the absence of any medicine, which was explicitly stated in the initial instructions as

117 well as apparent on several "no medicine" (n-) trials.

118 Note that medicines $\mathrm{C}$ and c' both occurred only in combination with one another, so

119 that target medicine C was perfectly confounded with its compound counterpart.

120 Medicine D also occurred only in combination with another medicine, e, however,

121 "elemental" medicine e also occurred by itself, allowing for an estimation of the

122 independent causal influence of medicine D across the constant presence of e (i.e., 
123 across e- and De+trials). Medicine S was identical to medicine $\mathrm{D}$, except that the

124 probability of headache given the Se compound was 0.5 rather than 1.0 ; a

125 comparison of medicines $S$ and $D$, therefore, identifies a difference between

126 deterministic and stochastic causation. Finally, medicine s' also produced headache

127 with a probability of 0.5 but unlike medicine $S$, never occurred in combination with

128 any other medicine, so that contrasting $S$ with $s^{\prime}$ identifies differences due to 129 compound presentation. From a modeling perspective, an algorithm that relies

130 solely on outcome variance to track uncertainty would only respond to medicines S

131 and s'. In contrast, a computation that treats both confounding and stochasticity as

132 sources of uncertainty should generate an increased signal to medicine $\mathrm{C}$ as well as

133 S and s': These divergent predictions are respectively instantiated by the error-

134 driven and Bayesian causal model specified in the subsequent section.

A.

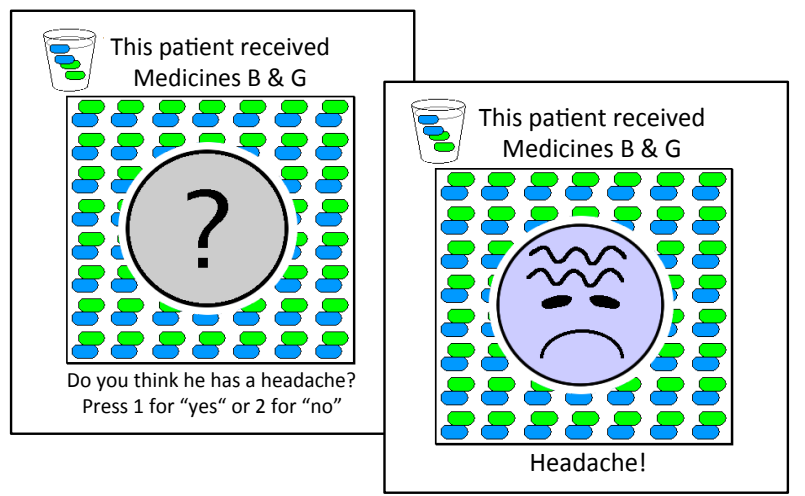

B.

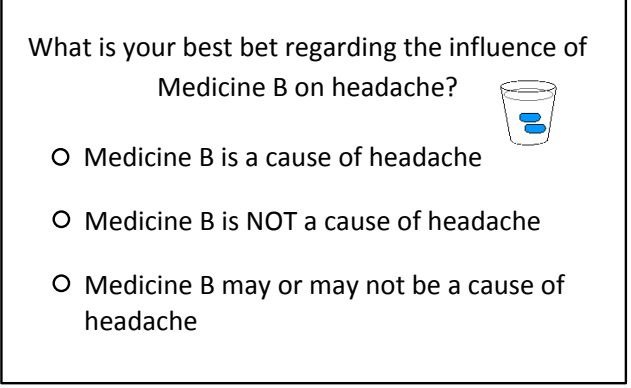

Figure 1. Task illustration. A. Prediction and outcome screens from a feedback-based learning trial. Screens were separated by a 2 sec. pause during which the word "Wait" (not shown in figure) was displayed. B. Causal query regarding the individual influence of a particular medicine on headache.

136 On each trial of feedback-based learning (see Figure 1A), participants were 137 presented with an individual allergy patient and were told either that the patient had 138 not received any medicine or that a particular medicine, or combination of 
139 medicines, had been administered. Medicines were color coded and labeled 140 accordingly (i.e., Medicine "B" was blue) with color assignments counterbalanced

141 across participants. On the first screen, the allergy patient's state was obscured by 142 a question mark and participants were asked to indicate whether or not the patient 143 had a headache, pressing the "Y" key for "yes" and the "N" key for "no". After a 144 prediction was made, there was a brief (2000ms) pause during which the word "wait" 145 was displayed on the screen, followed by a screen revealing the allergy patient's 146 state (i.e., with or without headache).

147 Each of 6 distinct trial types was presented 8 times in each of 4 sessions, for a total 148 of 192 trials, with sessions separated by 2-minute breaks, during which the scanner 149 was turned off. Each session was further divided into 4, non-delineated, blocks, with 150 each type of trial occurring once in random order in each block. All trials were 151 separated by a jittered 4-second ITI. In every other block, each trial (except for n152 trials) was followed by a query regarding the individual causal influence of the target 153 medicine present on that trial (i.e., C, D or S), or of medicine e or s' on non-target 154 trials. Specifically, participants were asked to choose between the following three 155 options regarding the relevant medicine: "is a cause of headache", "is not a cause 156 headache" and an ambivalent "may or may not be a cause of headache" (Figure 157 1B). Categorical response options, rather than the rating scales commonly 158 employed in the causal learning literature (Lu et al., 2008; Liljeholm \& Cheng, 2009; 159 Liljeholm, 2015) were used for two main reasons: First, because the limited number 160 of response buttons in the scanner would have necessitated a scale slider, the initial 
161 positioning of which might potentially bias ratings, and second, to reduce movement

162 due to back-and-forth scale scrolling.

163 Computational models. Two formal accounts of predictive strength and uncertainty

164 were implemented. First, an error-driven, associative learning algorithm updates 165 predictive strength, on each trial, using the difference between the observed state of 166 the outcome and the expected state based on all present cues:

$167 \quad V_{c, n+1} \leftarrow V_{c, n}+\alpha_{c, n}\left(\lambda-\sum_{i} V_{i}\right)_{n}$

168 where $V$ is the predictive strength of a particular cue, $c, \Sigma V_{i}$ is the summed strength of

169 all cues present on trial $n$, and $\lambda$ is the observed state of the outcome on that trial. The 170 associability of cue $c$ on trial $n, \alpha_{c, n}$, is defined as:

$171 \quad \alpha_{c, n}=\gamma\left|\lambda-\sum_{i} V_{i}\right|_{n-1}+(1-\gamma) a_{c, n-1}$

172 where $n-1$ references to the previous trial involving cue $c$, and $\gamma$ is a free parameter

173 accounting for the weighting of that previous trial relative to preceding ones. In 174 addition to scaling the influence of the prediction error on predictive strength, $\alpha$ is 175 taken to reflect current levels of uncertainty about the cue's influence (e.g., Esberg \& 176 Haselgrove, 2011). The strengths and uncertainties associated with each cue were

177 initialized to 0.5. On feedback-based trials with target causes, which always occurred 178 in compound with an alternative cause, the $\alpha$ of the target cause (always greater than 179 or equal to that of its compound counterpart) was used to indicate uncertainty, while 
180 the summed strength, $\Sigma V$, was used to predict the outcome, and to compute the 181 prediction error.

182 A second formal account is provided by a Bayesian Causal Model, in which reasoners 183 make inferences over causal structures potentially responsible for the observed data 184 (Griffiths \& Tenenbaum, 2005; Lu et al., 2008; Liljeholm, 2015). Here, as illustrated in 185 Figure 2, three possible graphs (Graphs 0-2) were defined for a candidate cause, $C$, an 186 alternative cause, $A$, and a constantly present background cause, $B$, where a causal 187 link to the effect exists for neither $C$ nor $A\left(G_{0}\right), C$ only $\left(G_{1}\right)$, or both $C$ and $A\left(G_{2}\right)$.
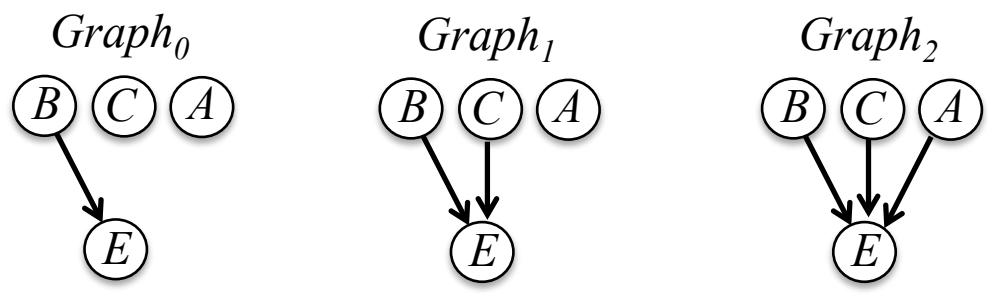

Figure 2. Possible causal structures involving a background cause, $B$, a target cause, $C$, an alternative cause, $A$, and an effect, $E$. A link to the effect always exists for $B$, and

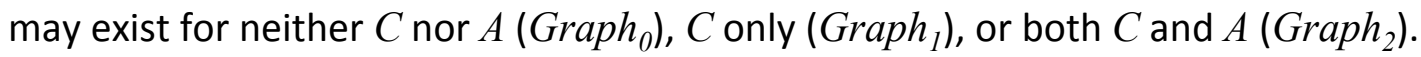

Each graph has a set of parameters $\theta$, which are strengths $w_{i}$ associated with causal links in each graph (i.e., $w_{B}, w_{C}$ and $w_{A}$ for links associated with $B, C$ and $A$ respectively

191 in $G_{2}$ ). Sequential estimates of these parameters were modeled, for each candidate 192 cause, using the smallest possible focal set (Cheng \& Novick, 1992) - a set of events

193 across which alternative causes can be assumed to occur with the same probability in 194 the presence and absence of the relevant cause. Thus, estimates of the strength of 195 the "no medicine" background cause was modeled using only n- trials in $G_{0}$. Estimates 196 of elemental (non-target) candidate causes s' and e were respectively modeled based 197 on trials with those causes and the "no medicine" trials (i.e., n- and s' \pm for candidate 
198 s', and n- and e- for candidate e), using $G_{l}$. Finally, for each target candidate cause,

199 which always occurred in compound with some alternative cause, estimation was 200 modeled given $G_{2}$, using the trials relevant for the particular target cause (i.e., n- and 201 Cc' for target C; n-, e- and De+ for target D, and n-, e- and Se \pm for target S).

202 The likelihoods $P\left(d \mid \theta, G_{i}\right)$ were computed using a noisy-OR parameterization (Cheng, 203 1997). Specifically, summarizing data $d$ by contingencies $N(e, c, a)$, the frequencies of 204 each combination of the presence versus absence of the effect, target cause and 205 alternative cause, the likelihood term for $G_{2}$ is:

$P\left(d \mid w_{B}, w_{C}, w_{A}, G_{2}\right)=\left(\begin{array}{c}N\left(c^{-}, a^{-}\right) \\ N\left(e^{+}, c^{-}, a^{-}\right)\end{array}\right) \times\left(\begin{array}{c}N\left(c^{+}, a^{-}\right) \\ N\left(e^{+}, c^{+}, a^{-}\right)\end{array}\right) \times\left(\begin{array}{c}N\left(c^{-}, a^{+}\right) \\ N\left(e^{+}, c^{-}, a^{+}\right)\end{array}\right) \times\left(\begin{array}{c}N\left(c^{+}, a^{+}\right) \\ N\left(e^{+}, c^{+}, a^{+}\right)\end{array}\right) w_{B}^{N\left(e^{+}, c^{-}, a^{-}\right)}$

$\left(1-w_{B}\right)^{N\left(e^{-}, c^{-}, a^{-}\right)}\left[w_{B}+w_{C}-w_{B} w_{C}\right]^{N\left(e^{+}, c^{+}, a^{-}\right)}\left[1-w_{B}-w_{C}+w_{B} w_{C}\right]^{N\left(e^{-}, c^{+}, a^{-}\right)}\left[w_{B}+w_{A}-w_{B} w_{A}\right]^{N\left(e^{+}, c^{-}, a^{+}\right)}\left[1-w_{B}-w_{A}+w_{B} w_{A}\right]^{N\left(e^{-}, c^{-}, a^{+}\right)}$

$\left[w_{B}+w_{C}+w_{A}-w_{B} w_{C}-w_{B} w_{A}-w_{C} w_{A}+w_{B} w_{C} w_{A}\right]^{N\left(e^{+}, c^{+}, a^{+}\right)}\left[1-w_{B}-w_{C}-w_{A}+w_{B} w_{C}+w_{B} w_{A}+w_{C} w_{A}-w_{B} w_{C} w_{A}\right]^{N\left(e^{-}, c^{+}, a^{+}\right)}$.

207 where $\left(\begin{array}{l}n \\ k\end{array}\right)$ denotes the number of ways of picking $k$ unordered outcomes from $n$ 208 possibilities. $N\left(c^{+}\right)$indicates the frequency of events in which the target cause is 209 present, with analogous definitions for the other $N($.$) terms. The likelihood terms for G_{0}$ 210 and $G_{1}$ are similarly specified, where frequencies $N($.$) are summed across the$ 211 presence and absence of relevant events, and $w_{i}=0$ for any cause that does not have 212 link to the effect in the relevant graph.

213 The marginal posterior distribution over strengths for a particular cause is obtained by 214 applying Bayes' rule, and integrating out the parameters for other causes in the graph, 215 such that, for $G_{2}$, 
$216 P\left(w_{C} \mid d, G_{2}\right)=\int_{0}^{1} \int_{0}^{1} \frac{P\left(d \mid w_{B}, w_{C}, w_{A}, G_{2}\right) P\left(w_{B}, w_{C}, w_{A} \mid G_{2}\right)}{P(d)} d w_{B} d w_{A}$,

217 where $P\left(d \mid w_{B}, w_{C}, w_{A}, G_{2}\right)$ is the likelihood term, $P\left(w_{B}, w_{C}, w_{A} \mid G_{2}\right)$ refers to the prior

218 probabilities of causal strength parameters, and $P(d)$ is the normalizing term, denoting 219 the probability of the observed data.

220 Uncertainty about the strength of a particular cause, the focus of the study, was

221 modeled as the Shannon entropy $H\left(w_{c}\right)$ of its marginal posterior distribution $P\left(w_{C} \mid d\right)$ :

$222 \quad H\left(w_{C}\right)=-\int_{0}^{1} P\left(w_{C} \mid d\right) \ln P\left(w_{C} \mid d\right) d w_{C}$.

223 Point estimates of causal strength for each elemental and target cause were modeled

224 as the mean of the relevant marginal posterior distribution (Lu et al., 2008; Liljeholm, 225 2015), given by:

226

$\bar{w}_{C}=\int_{0}^{1} w_{C} P\left(w_{C} \mid d\right) d w_{C}$

227 The model was sequentially implemented on a trial-by-trial basis, such that, for each

228 cause, likelihoods were computed on each trial of feedback-based learning that 229 yielded information relevant to that cause, using only the data point provided on that 230 trial, with the posterior on the previous trial being used as the prior to obtain the 231 posterior on the current trial. On the first trial in which data relevant to a particular 232 candidate cause was presented, the priors were assigned independent uniform 233 distributions. Finally, as an analog to the model-free prediction error, the Kullback- 
234 Leibler $(\mathrm{KL})$ divergence between the prior $(Q)$ and posterior $(P)$ on each trial,

235 commonly referred to as "Bayesian surprise" (Itti \& Baldi, 2006) was computed as:

$$
K L(P \| Q)=\int_{0}^{1} p\left(w_{C} \mid d\right) \log \frac{p\left(w_{C} \mid d\right)}{q\left(w_{C} \mid d\right)} d w_{C} .
$$

237 As noted, target causes only occurred in compound with alternative causes during 238 feedback-based learning. On such trials, the entropy and $\mathrm{KL}$ divergence of the 239 marginal distribution for the target cause was used to indicate uncertainty and surprise 240 respectively, while predictions regarding the occurrence of the effect were generated 241 using a noisy-OR integration of causal strengths.

242 Both models assumed that, during feedback-based learning, participants selected 243 "Yes"/"No" responses to questions about whether headache would occur using 244 probabilities generated by a softmax distribution, in which a free noise parameter 245 controls the influence of predictive strength (i.e., the strength with which the 246 medicine(s) present on a given trail predicted headache; noisy-logical $P\left(e \mid w_{i}\right)$ and $\Sigma V$ 247 in causal and error-driven models respectively) on choice behavior. An additional 248 noise parameter controlled the influence of uncertainty (i.e., $\alpha$ and $H\left(w_{c}\right)$ in error249 driven and causal models respectively) on the proportion of ambivalent "May or may 250 not be a cause" judgments during queries about the individual influence of each 251 medicine. The first two instances of each trial type, and the first causal judgment for 252 each medicine, were excluded from model fitting to eliminate transient noise due to 253 task adjustment, and to minimize the influence of assumptions about priors. Free 254 parameters were fit to behavioral data by minimizing the negative log likelihood of 
255 observed responses for each individual and the Bayesian Information Criterion (BIC) 256 was used for model selection.

257 The model-derived probabilities of uncertain "May or may not be a cause" judgments

258 are plotted in Figure 3 for the causal and error-driven account of uncertainty 259 respectively. Note that, as was foreshadowed in the description of the study design, 260 the Bayesian causal model predicts high levels of uncertainty for both confounded, C, 261 and stochastic, S and s', causes, while the error-driven algorithm predicts that only 262 stochasticity will generate high levels of uncertainty.

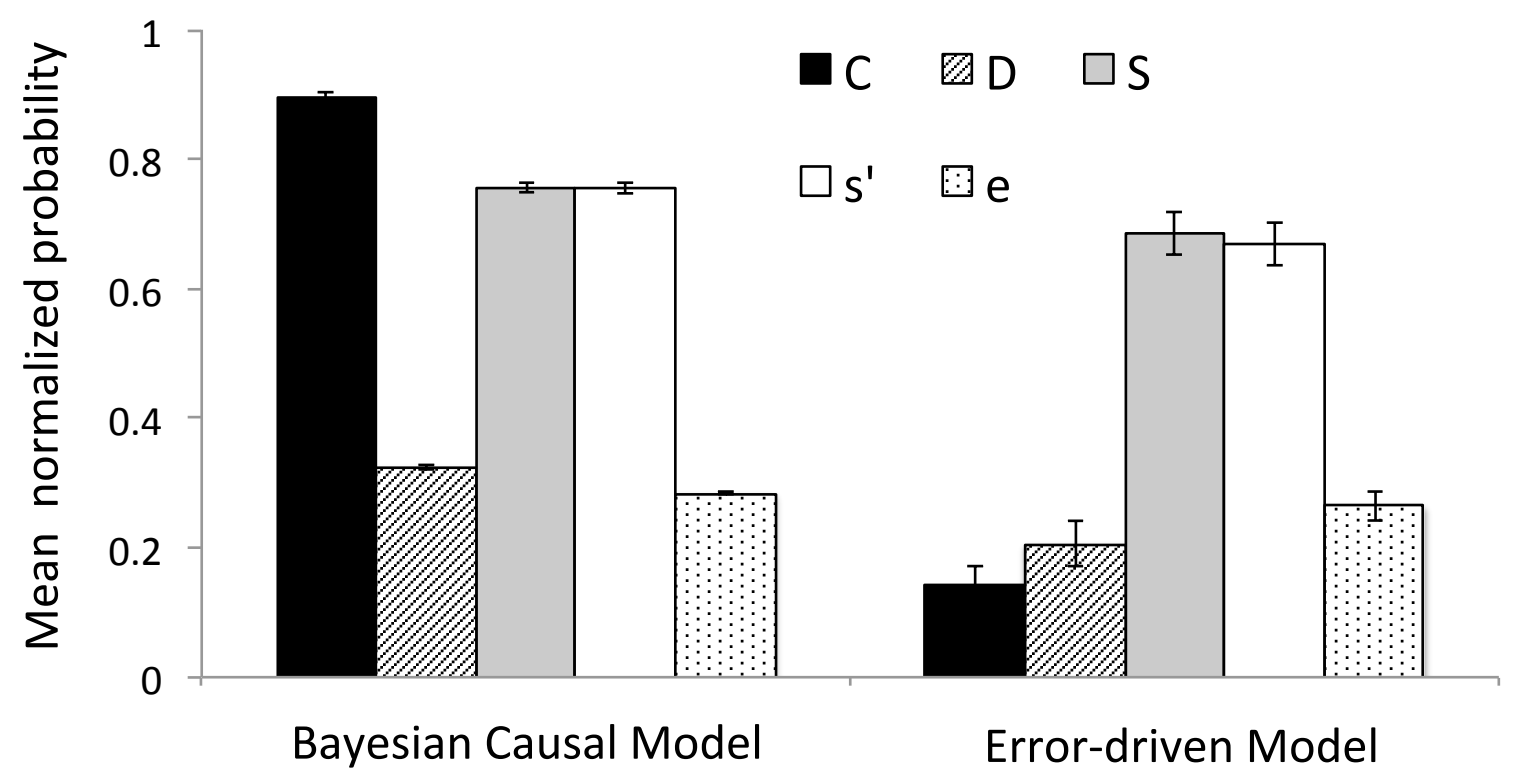

Figure 3. Mean (normalized) probabilities of uncertain "May or may not be a cause" judgments derived from the Bayesian causal model and error-driven algorithm respectively. Confounded, deterministic and stochastic target causes (C, D \& S) occurred only in compound with alternative causes during feedbackbased learning, while non-target causes occurred only individually ( $\left.s^{\prime}\right)$, or individually as well as in compound (e). Error bars=SEM. 
264 Imaging procedure and analysis. A 3 Tesla scanner (Phillips Achieva) was used to 265 acquire structural T1-weighted images and T2*-weighted echoplanar images

266 (repetition time $=2.65 \mathrm{~s} ;$ echo time $=30 \mathrm{~ms} ;$ flip angle $=90^{\circ} ; 45$ transverse slices; 267 matrix $=64 \times 64$; field of view $=192 \mathrm{~mm}$; thickness $=3 \mathrm{~mm}$; slice gap $=0 \mathrm{~mm}$ ) with 268 BOLD contrast. The first four volumes of images were discarded to avoid T1 269 equilibrium effects. All remaining volumes were corrected for differences in the time of 270 slice acquisition, realigned to the first volume, spatially normalized to the Montreal 271 Neurological Institute (MNI) echoplanar imaging template, and spatially smoothed with 272 a Gaussian kernel (8 $\mathrm{mm}$, full width at half-maximum). High-pass filtering with a cutoff 273 of 128 s was used. All effects were reported at a whole brain FWE cluster corrected 274 threshold of $p<0.05$, calculated using the Statistical nonParametric Mapping toolbox 275 (SnPM13; http://warwick.ac.uk/snpm; Nichols \& Holmes, 2002), with 5000 276 permutations, no variance smoothing and an uncorrected height threshold of $p<$ $277 \quad 0.001$.

278 To address collinearity due to the shared prediction by causal and error-driven 279 accounts that uncertainty increases with increased stochasticity, the two algorithms 280 were analyzed using separate $1^{\text {st }}$ level General Linear Models (GLM), and were 281 directly contrasted at the group level using Bayesian Model Selection. Specifically, for 282 each participant, two GLMs were specified, one for the causal and one for the error283 driven computational model. In each such GLM, three regressors respectively 284 modeled 1) the onset of the prediction screen on each trial during feedback-based 285 learning, 2) the onset of the outcome screen on each trial during feedback-based 286 learning, and 3) the onsets of the screens soliciting judgments about the influences of 
287 individual medicines. In one GLM, prediction-screen onsets and causal judgment 288 onsets were modulated by trial-by-trial error-driven estimates of uncertainty and 289 strength, while outcome-screen onsets were modulated by trial-by-trial absolute 290 values of the prediction errors. In the second GLM, prediction-screen and causal 291 judgment onsets were modulated by trial-by-trial estimates of uncertainty and strength 292 by the causal model while outcome-screen onsets were modulated by trial-by-trial 293 estimates of Bayesian surprise. In both models, the first two instances of each trial 294 type, and corollary causal judgments, were modeled by separate regressors, to 295 eliminate transient noise due to task adjustment and reduce the influence of, non296 modeled, prior beliefs. Moreover, in each model, two onset regressors respectively 297 modeled the responses on feedback-based and causal query trials, three regressors 298 indicated separate sessions, and six additional regressors accounted for the residual 299 effects of head motion. Except for those indicating sessions and head motion, all 300 regressors were convolved with a canonical hemodynamic response function. No 301 orthogonalization was applied.

302 To directly contrast error-driven and causal accounts, BMS analyses was performed 303 using a set of GLMs with the same onsets as those specified above, but with a single 304 parametric modulator (e.g., error-driven uncertainty at prediction screen onsets) 305 entered for each GLM. Thus, model comparisons isolated the relative contribution of 306 a particular computational variable to neural activity. First-level Bayesian estimation 307 procedure was used to compute a log-model evidence map for every subject and 308 each GLM and inferences at the group level were modeled by applying a random 309 effects approach (Rosa et al., 2010) at every voxel of the log evidence data. Group- 
310 level exceedance probability maps, reflecting the probability that one model is more

311 likely than the other, were thresholded to identify voxels in which the exceedance

312 probability was greater than 0.95. Classical inferences were then assessed using

313 BMS masks, within which a particular computational variable was more likely than its

314 competing counterpart, at an exceedance probability greater than 0.95: For example,

315 significant effects of error-driven uncertainty during the prediction period of feedback-

316 based learning trials were reported only for those regions in which error-driven

317 uncertainty during this event period was more likely than causal uncertainty during the

318 same period, according to the BMS.

319 A pilot study on error-driven uncertainty. Previous research has implicated the 320 anterior insula and dorsal medial frontal cortex in outcome stochasticity, using a range 321 of decision-making tasks (Mohr et al., 2010; Volz et al., 2003; Huettel et al., 2005;

322 Grinband et al., 2006). To ensure that such effects, used here as a benchmark 323 against which to assess neural representations of uncertainty due to confounding, 324 also emerge in our causal learning task, a pilot study $(n=10)$ was conducted that was 325 highly similar to that reported here, with the following exceptions: First, only 326 stochasticity, not confounding, was manipulated across putative causes, second, both 327 generative and preventive causal influences were included and, finally, judgments of 328 individual causal influences were solicited at the end of, rather than throughout, each 329 scanning session, and measured on a scale raging from -100 (strongly removes 330 headache) to +100 (strongly produces headache). The error-driven model of 331 uncertainty was implemented as described above, fit to behavior during feedback332 based learning, and regressed against the BOLD data. A single region of interest 
333 (ROI) was constructed from the anterior insula and dorsal medial frontal cortex, using

334 the "Willard" functional parcellation atlas (Richiardi et al., 2015). A power analysis

335 performed with NeuroPower (http://neuropowertools.org; Durnez et al., 2015), using a 336 screening threshold of $z=2.3$, revealed that a sample size of 16 would yield a power of 3370.9 to detect effects of error-driven predictive uncertainty in this ROI at a Gaussian 338 random field corrected alpha level of 0.05 .

340 Results

\section{Behavioral results}

342 All statistical tests of behavioral data were planned comparisons, employing two-tailed 343 t-tests, and were calculated using $n=19$. Confidence intervals $(C /)$ and effect sizes 344 (Cohen's $d$ ) are reported for all comparisons. The distributions of causal judgments 345 across response options are shown, with associated response times, for each putative 346 cause in Table 1. Note that the proportion of ambivalent "May or may not be a cause" 347 judgments $\left(3^{\text {rd }}\right.$ row in Table 1$)$ were greatest for the confounded cause $(C)$, 348 intermediate, although still substantial, for the two stochastic causes ( $S$ and s'), and 349 virtually absent for deterministic causes ( $D$ and e), exactly as predicted by the causal, 350 but not the error-driven, model (cfr Figure 3; see Liljeholm, 2015 for similar results).

351 Note also that the distribution of "Is a cause" and "Is not a cause" judgments differed 352 markedly across stochastic and confounded causes, such that participants almost 353 never select the latter option for the confounded cause, consistent with the normative 354 increase in the likelihood that target cause $\mathrm{C}$ is causal given $\mathrm{Cc}+$ trials, but distributed 
355 their responses fairly evenly across these two options for stochastic causes, reflecting,

356 perhaps, the outcome on trials immediately preceding each judgment.

357 Planned comparisons revealed that the mean proportion of ambivalent causal 358 judgments were significantly lower for the deterministic (D) target cause than for both 359 confounded $(C), t(18)=6.78, p<0.001, C l=\left[\begin{array}{ll}0.35 & 0.67\end{array}\right], d=1.79$, and stochastic (S), $360 \mathrm{t}(18)=4.09, p<0.001, C l=[0.140 .44], d=1.11$, target causes. The difference between 361 the confounded and stochastic cause was also significant, with the mean proportion of 362 ambivalent judgments being greater for $C$ than for $S t(18)=2.65, p<0.05, C l=[0.0 .05$ $3630.40], d=0.68$. Recall that all target causes, including $S$, occurred only in compound 364 with alternative causes during feedback-based learning. The proportion of ambivalent 365 judgments for the two stochastic causes, one always occurring in isolation (s') and the 366 other always in compound (S), was marginally significant, $p=0.08$.

367 Judgment response times, averaged across response options for each cause, were 368 significantly faster for judgments about the deterministic target cause $D$ than 369 stochastic target cause $\mathrm{S}, \mathrm{t}(18)=2.20, p<0.05, C l=[0.031 .02], d=0.35$, but did not differ 370 significantly between $D$ and $C$, nor between $C$ and $S, p>0.1$. Moreover, response 371 times did differ significantly across the two stochastic causes, being significantly 372 slower for target cause $\mathrm{S}$, which never occurred in isolation during feedback-based 373 learning, than for cause $s^{\prime}, \mathrm{t}(18)=3.42, p<0.005, C l=[0.291 .21], d=0.52$.

374 With respect to model fitting, the mean best fitting values of the free parameters for 375 the error driven model were $0.35 \pm 0.27$ for the weighting parameter, $3.25 \pm 0.95$ for the 376 prediction noise parameter, and $1.08 \pm 0.88$ for the judgment noise parameter. The 
377 mean best fitting values of the free parameters for the causal model were $4.13 \pm 1.44$

378 for the prediction noise parameter and $1.03 \pm 1.05$ for the judgment noise parameter.

379 The mean BIC was significantly lower, indicating superior performance, for the causal 380 model $(220.00 \pm 44.15)$ than for the error-driven model $(237.09 \pm 35.58), t(18)=2.51$, $381 p<0.05, C l=[2.8131 .37], d=0.43$.

\section{$382 \quad$ Neuroimaging results}

383 All results reported below survived inclusive masking with voxels identified by

384 Bayesian model selection analyses. A complete list of the non-masked significant 385 effects of each model is provided in Tables 2 and 3. Bar graphs show effect sizes 386 extracted from $8 \mathrm{~mm}$ spheres centered on peak MNI coordinates, using rfxplot 387 (Glascher, 2009).

388 Uncertainty signals during causal judgments. During causal queries, as participants 389 made judgments about the causal influences of individual medicines, selective and 390 significant effects of the causal model of uncertainty emerged in the medial and lateral 391 superior frontal gyrus and the right dorsolateral prefrontal cortex (Figure 4). Note that 392 the effect size of BOLD responses (bar graphs in Figure 4) across putative causes 393 were greatest for the confounded cause (C), intermediate for stochastic causes (S and 394 s'), and smallest for deterministic causes ( $D$ and e), exactly as predicted by the 395 causal, but not the error-driven, model (cfr Figure 3). To further probe these effects, 396 response times (RTs) were included as a parametric modulator of judgment 397 uncertainty in $1^{\text {st }}$ level, individual-subject, models, and as a between-subjects 398 covariate in group-level analyses. Neither of these additional analyses revealed any 
399 modulatory influence by RTs on the neural effects of the causal model of judgment 400 uncertainty. No effects of the error-driven measure of uncertainty during causal 401 judgments survived whole-brain correction.
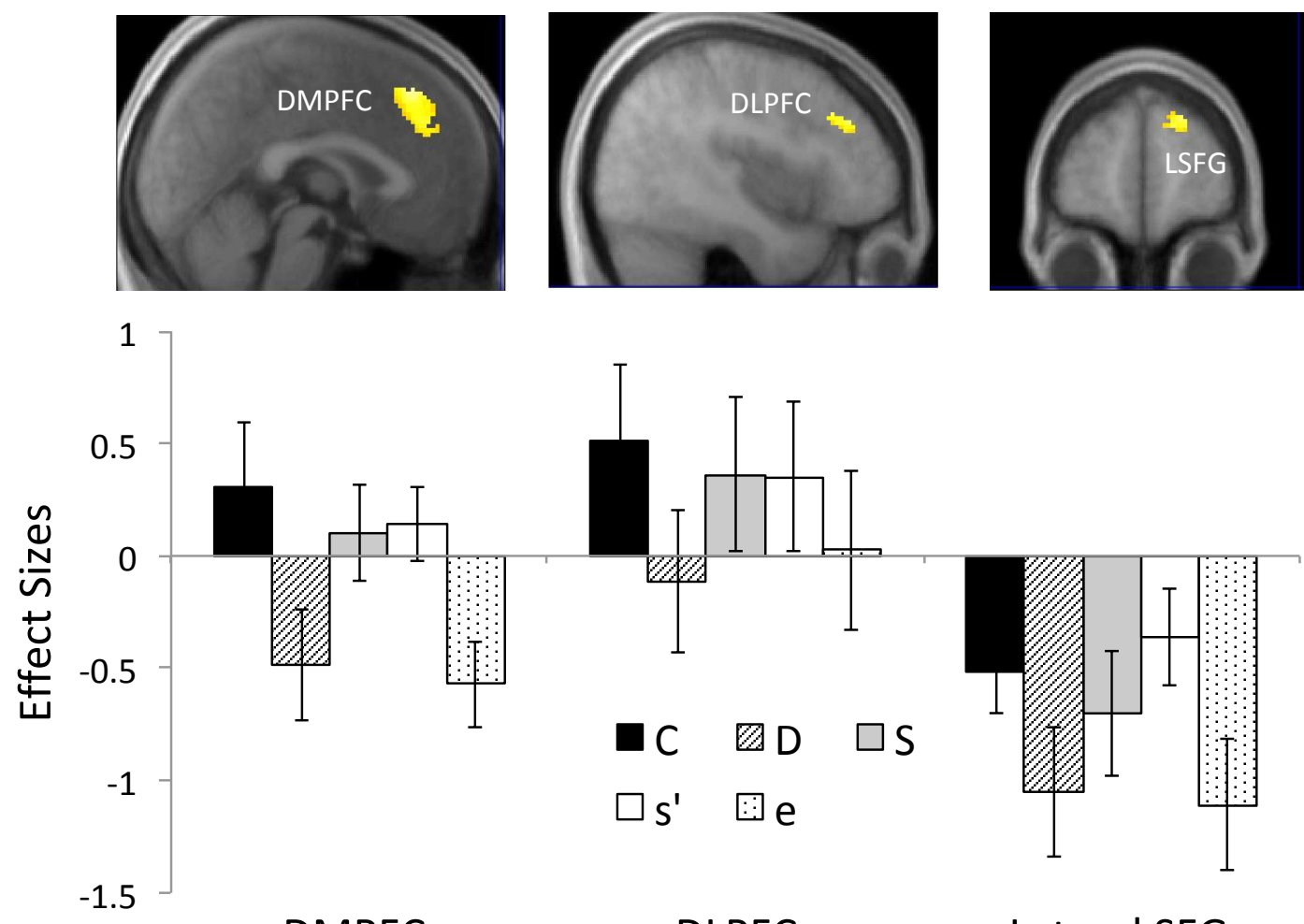

DMPFC
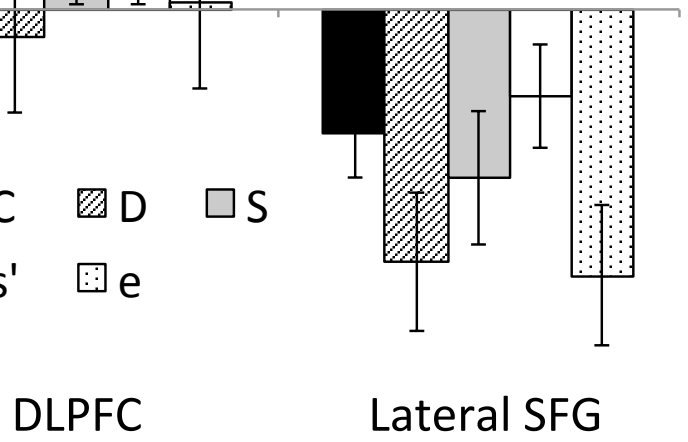

Figure 4. Selective effects of the causal model of uncertainty during judgments about the causal influences of individual medicines, showing effects in the dorsomedial and dorsolateral prefrontal cortex (DMPFC and DLPFC), and the lateral Superior Frontal Gyrus (LSFG). Bar plots show effect sizes extracted from spheres centered on peak coordinates for each queried medicine. Error bars=SEM.

403 Uncertainty signals during prediction. During the prediction period of feedback-based 404 learning trials, the error-driven, but not causal, measure of uncertainty was 405 significantly positively correlated with activity throughout the inferior occipital and 406 lingual gyri, as well as with activity in the supplementary motor area and right anterior 
407 insula (Figure 5). Moreover, activity negatively correlated with error-driven predictive 408 uncertainty was observed in the posterior middle temporal gyrus.
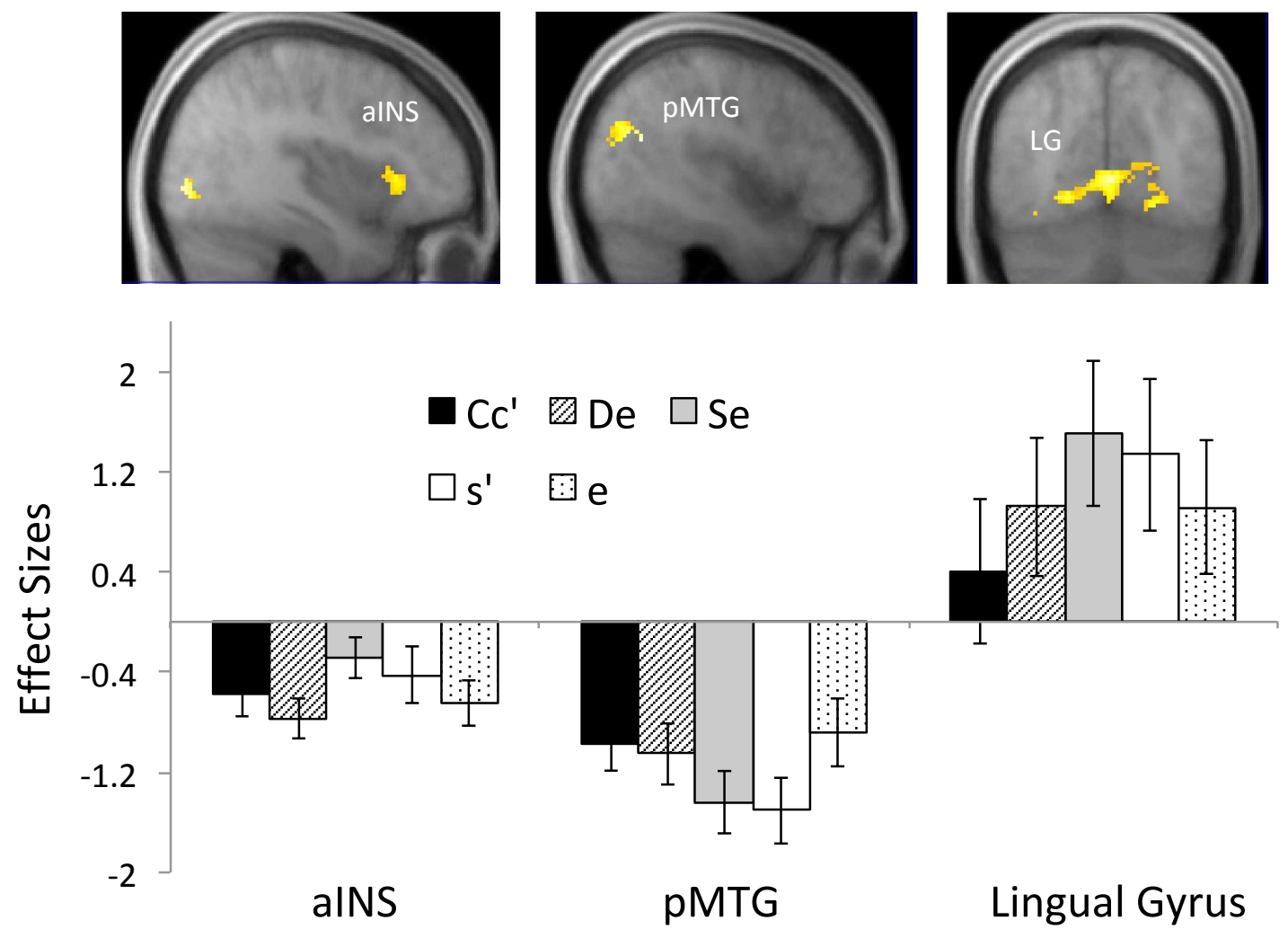

Figure 5. Selective effects of the error-driven model of uncertainty during the prediction period of feedback-based learning trials, showing effects in the right anterior insula (aINS), posterior middle temporal gyrus (pMTG) and lingual gyrus (LG). Bar plots show effect sizes extracted from spheres centered on peak coordinates for each type of medicine trial. Error bars=SEM.

410 These effects of the error-driven account of uncertainty appear relatively robust, as

411 they closely mirror those from the pilot study, in which, at an uncorrected threshold of 4120.005 , extensive (cluster size > 100) effects of error-driven predictive uncertainty 413 emerged in the dorsal medial frontal cortex, bilateral anterior insula, and lingual gyrus 
14 during feedback-based learning. In contrast to the widespread effects of error-driven

415 predictive uncertainty during feedback-based learning, selective and significant effects

416 of the causal measure of uncertainty emerged only in the right dorsal thalamus.

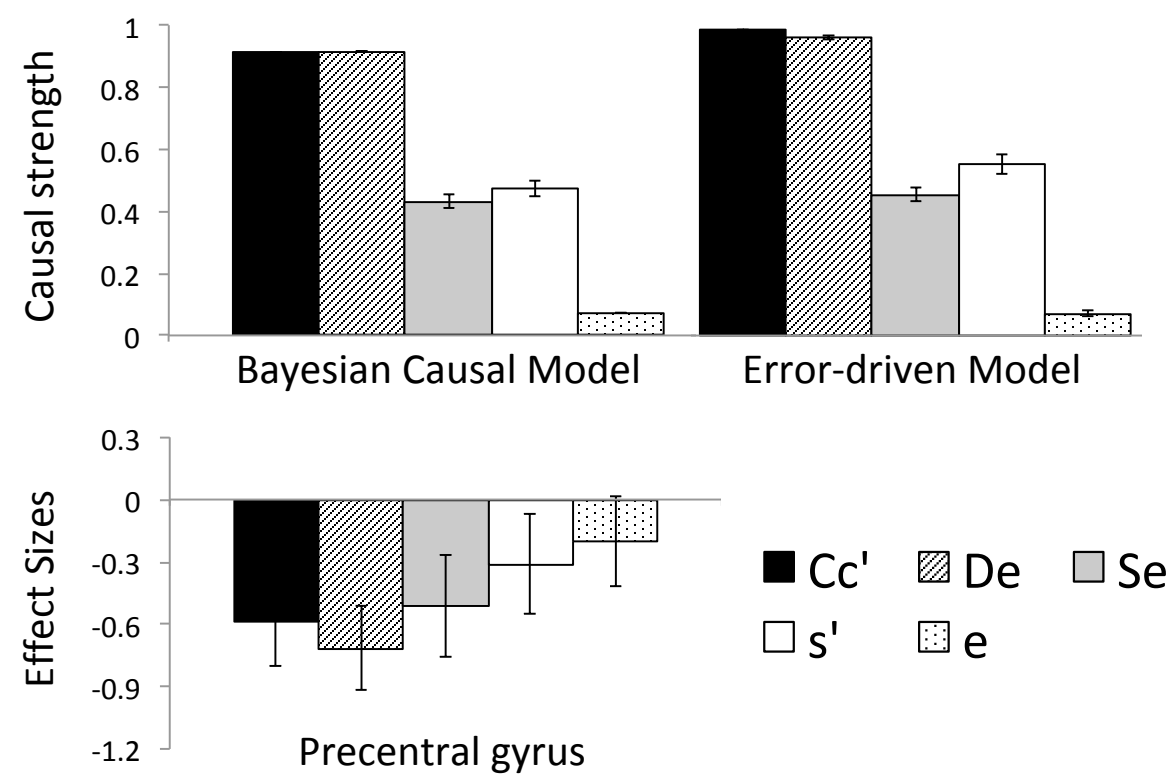

Figure 6. Strength predictions derived from the causal and error-driven model, together with effect sizes extracted from spheres centered on peak coordinates in the precentral gyrus, for each type of medicine trial during feedback-based learning. Error bars=SEM.

418 Strength \& surprise signals. While uncertainty in causal inferences, due to 419 confounding or stochasticity, is of primary interest here, estimates of the strength with 420 which a cause, or combination of causes, predicts the effect, and of the surprise at the 421 outcome on each trial of feedback-based learning, were also modeled for 422 completeness. During the prediction period of feedback-based learning trials, the 423 causal measure of predictive strength was selectively and significantly positively 424 correlated with activity in the calcarine and superior occipital gyrus, as well as 
425 negatively correlated with activity in the left lateral superior frontal and precentral gyri

426 (Figure 6). In contrast, a selective and significant negative correlation with the error-

427 driven measure of predictive strength emerged in the posterior MTG. No significant 428 effects of either the causal or error-driven account of predictive strength were found 429 during causal judgments.
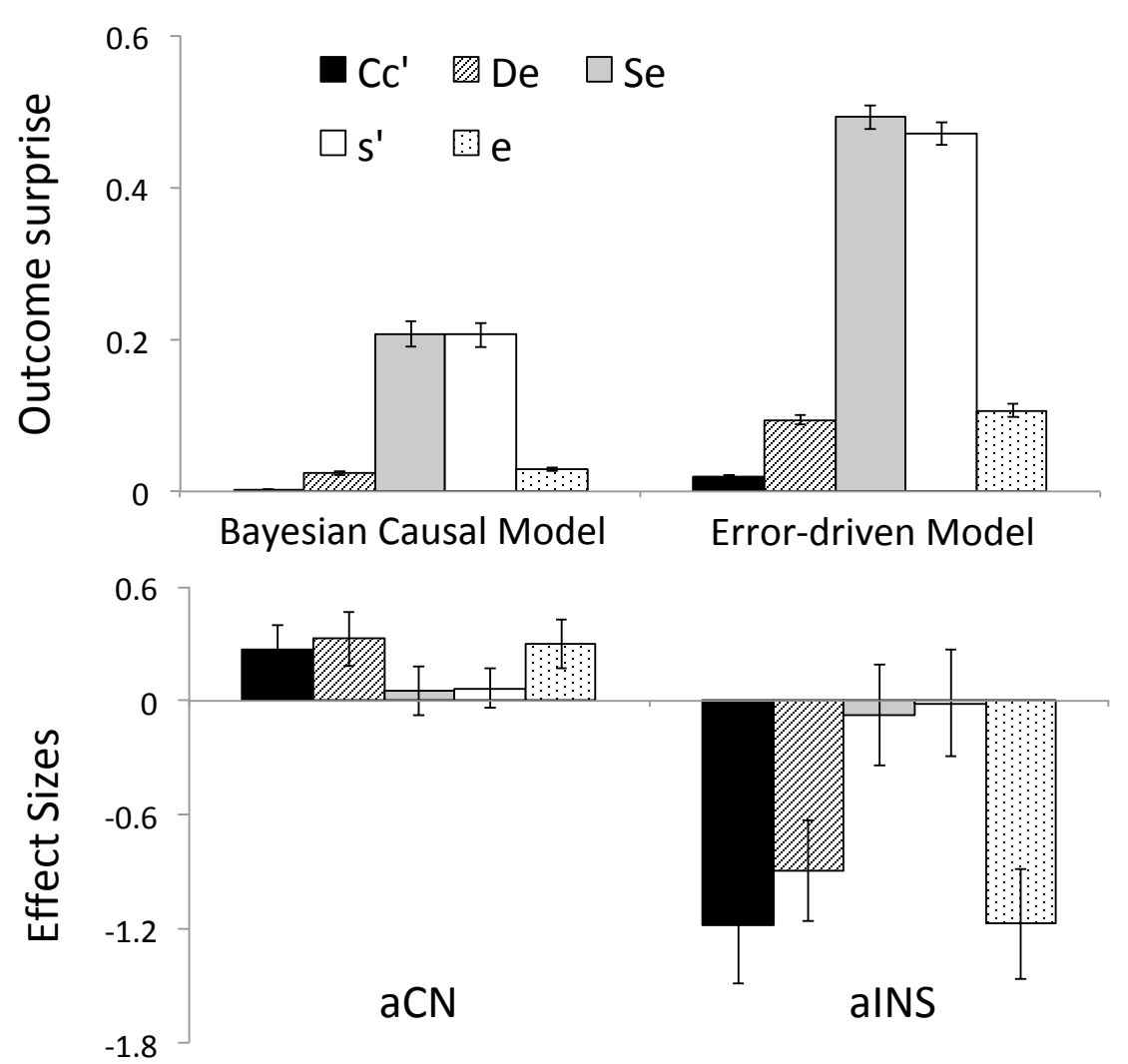

Figure 7. Outcome surprise signals derived from the causal and errordriven model (top), together with effect sizes (bottom) extracted from spheres centered on peak coordinates in the anterior caudate nucleus $(\mathrm{aCN})$ and anterior insula (aINS), for each type of medicine trial during feedback-based learning. Error bars=SEM.

431 For measures of surprise during the outcome period of feedback-based learning trials, 432 the absolute prediction error generated by the error-driven account was selectively 
433 and significantly positively correlated with activity in the bilateral anterior insula, the

434 medial and lateral dorsal prefrontal cortex and the inferior parietal gyrus. In addition,

435 significant negative correlations with this measure were identified in the middle 436 occipital gyrus, fusiform gyrus, the superior parietal lobule, precentral gyrus and 437 calcarine sulcus. In contrast, a selective and significant negative correlation with the 438 causal measure of (Bayesian) surprise was observed in the anterior caudate.

\section{Discussion}

440 Sensitivity to the "no confounding" constraint - a requirement that putative causes do 441 not co-vary, fundamental to philosophical and statistical perspectives on causation 442 has been reliably demonstrated in a range of behavioral judgments by children as well 143 as adults (Spellman, 1996; Kushnir and Gopnik, 2005; Meder et al., 2006; Schulz and 444 Bonawitz, 2007; Schulz et al., 2007; Liljeholm, 2015). The current study used fMRI 445 and cognitive computational modeling to investigate neural substrates mediating 446 uncertainty associated with causal confounding. While being scanned, participants 447 studied and judged the influences of various putative causes with confounded or non448 confounded, deterministic or stochastic, influences. During judgments requiring 449 generalization of causal knowledge from a feedback-based learning context to a 450 transfer probe, activity in the dorsomedial prefrontal cortex (DMPFC) was better 451 accounted for by a Bayesian causal model, sensitive to both confounding and 452 stochasticity, than a purely error-driven algorithm, sensitive only to stochasticity.

453 The DMPFC has been implicated in encoding uncertainty across a variety of tasks 454 (Volz et al., 2003; Grinband et al., 2006; Xue et al., 2008; Michael et al., 2015). For 455 example, scanning participants with $\mathrm{fMRI}$ as they classified stimuli according to a 
456 variable category boundary, Grinband et al. (2006) found that activity in the DMPFC 457 scaled with the proximity of a stimulus to a category boundary. An important aspect of 458 this and other demonstrations of the involvement of the DMPFC in uncertainty is that 459 the level of uncertainty is directly related to the degree of experienced errors: that is, 460 the greater the uncertainty on a given trial, the more likely it is that the prediction on 461 that trial will be incorrect, as indicated by explicit feedback. In contrast, during 462 feedback-based learning in the current task, the outcome on confounded trials was 463 deterministic. Consequently, the increased activity in the DMPFC, as participants 464 generated judgments about confounded cause, cannot be attributed to a history of 465 errors. Instead, these results suggest that the DMPFC encodes a more abstract 466 representation of uncertainty, divorced from the immediate consequences of choice, 467 and irrespective of whether the specific source of uncertainty is confounding or 468 stochasticity.

469 Several neuroeconomic studies (Hsu et al., 2005; Krain et al., 2006; Huettel et al., 470 2006; Bach et al., 2009; Levy et al., 2010; Pushkarskaya et al., 2015) have contrasted 471 gambles involving stochasticity with "ambiguous" gambles, for which outcome 472 probabilities are completely unknown, with some finding greater activity in the DMPFC 473 in response to ambiguity than stochasticity (e.g., Hsu et al., 2005). One might argue 474 that, in the current study, the influence of the confounded medicine is unknown, just 475 as the outcome probabilities of ambiguous gambles in previous studies were 476 unknown. But what does it mean to "know" something? Critically, all target medicines 477 occurred only in compound with alternative medicines during feedback-based 478 learning: consequently, when presented individually during causal queries, each was 
479 equally novel and, presumably, equally unknown. In other words, differences in 480 ambiguity between confounded and other target causes cannot be attributed to a 481 generalization decrement based on changes in stimulus features. Moreover, ambiguity 482 due to small samples and uncertainty due to confounding call for very different plans 483 of exploratory action: while in former case, repeated observation of the same stimulus 484 can resolve the uncertainty, the latter case requires an intervention that unconfounds 485 the stimulus configuration. Further work is needed to assess the overlap between 486 neural representations of confounding and ambiguity.

487 In addition to stochasticity and ambiguity, the DMPFC has been heavily implicated in 488 cognitive control (Ridderinkhof et al., 2004; Fellows \& Farah, 2005; Egner, 2009; 489 Taren et al., 2011; Shenhav et al., 2013), raising the question of whether the currently 490 observed effects in this region might reflect similar processes. In particular, 491 judgments about individual causal influences may be associated with efforts to 492 retrieve instances of the queried cause occurring in isolation from episodic memory 493 a search that is futile, and thus presumably more effortful, for confounded causes. 494 However, such retrieval-induced control demands are unlikely to account for the 495 current effects in the DMPFC for several reasons: First, effort associated with episodic 496 memory retrieval does not address responses to stochasticity - in contrast, the causal 497 uncertainty account explains why DMPFC responses are greater for both confounded 498 and stochastic causes, relative to non-confounded and deterministic causes. It may 499 be argued, of course, that stochasticity elicits additional control processes mediated ¡00 by the DMPFC, such as, for example, response conflict (Botnivick et al., 2001; j01 Ridderinkhof et al., 2004; Kerns et al., 2004). Recall, however that none of the target 
502 causes (C, S and D) were presented in isolation during feedback-based learning and ¡03 that, consequently, all target causes should involve some level of searching for non¡04 presented information during causal judgments. If DMPFC activity reflected this j05 search process as well as response conflict due to stochasticity, one would expect a ¡06 difference between neural responses to causes $S$ and s', both of which are stochastic, ¡07 but only one of which, s', was presented in isolation during feedback-based learning, j08 eliminating the need to search for an un-experienced memory. Notably, while RTs ¡09 (Table 1), commonly used as a measure of cognitive load, are indeed consistent with 510 the notion of an increased control demand for cause S over s', the DMPFC responses 511 (Figure 4) are not. Finally, and perhaps most conclusively, the inclusion of RTs as a j12 parametric modulator of judgment uncertainty in $1^{\text {st }}$ level models, and as a covariate in j13 group analyses, did not have any impact on the neural effects of model-based j14 judgment uncertainty. Taken together, these aspects of the results make causal 515 uncertainty the more parsimonious explanation of the DMPFC effects observed here.

j16 As with the DMPFC and DLPFC, the anterior insula has been implicated in outcome j17 stochasticity, or "risk", in several previous neuroimaging studies (e.g., Volz et al., 518 2003; Huettel et al., 2005; d'Acremont et al., 2013; Preuschoff et al., 2008; Critchley et j19 al., 2001). Here, the dorsal anterior right insula was selectively recruited by the error¡20 driven model of uncertainty during the prediction period of feedback-based learning 521 trials. Plots of effect sizes for each type of trial (in Figure 5) suggest that these ¡22 responses were indeed driven by stochasticity-based uncertainty about the state of ¡23 the outcome on any given trial. Moreover, the fact that, unlike the DMPFC, activity in ¡24 the anterior insula did not scale with either an error-driven or causal measure of 
uncertainty during judgments about individual causal influences indicates that

j26 representations in this region pertain primarily to the immediate consequences of

¡27 choice, rather than generalizable knowledge. Consistent with this interpretation, the ¡28 bilateral anterior insula also responded selectively to stochastic conditions during the j29 outcome period of feedback-based learning trials, suggesting that this region is 530 recruited by both the anticipation and detection of errors. While, as noted, it is difficult ¡31 to discern based on the current results whether the unsigned error signals observed in j32 the anterior insula are Bayesian or model-free, they are consistent with a substantial 533 literature implicating this region in expectancy violations across a wide range of tasks ¡34 (Klein et al., 2007; Metereau et al., 2012; Allen et al., 2016; Bastin et al., 2016).

535 Selective effects of the error-driven account of predictive uncertainty were also 536 identified throughout large portions of the posterior lingual gyrus. In a recent ¡37 neuroimaging study, Causse et al., (2013) assessed decision making under 538 uncertainty using an aviation task in which participants had to either land a plane or 539 abort landing, given certain (100\% or $0 \%$ ) vs. uncertain $(50 \%)$ information regarding j40 landing conditions. Consistent with the current results, they found greater activity in ¡41 the posterior lingual gyrus during high than during low uncertainty conditions. Our j42 results are also consistent with those of Payzan-LeNestour et al. (2016), who found j43 that the lingual gyrus scaled specifically with uncertainty due to outcome stochasticity, 544 as opposed to uncertainty due to changes in the statistical structure of the j45 environment or due to ambiguity. Payzan-LeNestour et al. argued that the lingual j46 gyrus might not typically emerge in studies assessing outcome stochasticity because j47 those studies did not dissociate stochasticity from other uncertainty components. A 
548 similar case can be made here, that the inclusion of an alternative, generative, model

j49 that captures additional aspects of uncertainty may have increased sensitivity to 550 detect stochasticity-specific signals in the lingual gyrus.

551 An important aspect of the current study is the direct comparison of a Bayesian j52 inference model with an error-driven, model-free, algorithm. While not uncommon 553 (Courville et al, 2006; Hampton et al., 2006; Payzan-LeNestour \& Bossaerts, 2011; j54 Prevost et al., 2013), the contrasting of these quite different computational frameworks 555 somewhat complicates interpretation. In particular, it raises the question of whether j56 selective effects of the causal account of uncertainty indeed reflect sensitivity to 557 confounding, or instead some extraneous, perhaps incidental, feature of the different j58 modeling frameworks. Here, a significant clue is provided by neural and behavioral j59 responses to each queried putative cause, shown in Figure 4 \& Table 1 respectively, 560 which clearly correspond to the increased uncertainty associated with confounded and 561 stochastic causes predicted by the causal model (cfr Figure 3). It is worth noting that, j62 far from incidental, the failure of the error-driven account to predict sensitivity to j63 confounding is intrinsic to the model-free approach, due to its lack of a representation 564 of the independence of causal influences. Critically, a configural solution (e.g., j65 Pearce, 1987; 2002), such that individually queried causes are treated as nonj66 overlapping with the compounds in which they occur during feedback-based learning, j67 would fail to account for both behavioral and neural differences between confounded j68 and deterministic target causes, both of which occurred only in compound with j69 alternative causes during feedback-based learning. 
570 While the effects of causal uncertainty in the DMPFC likely reflect sensitivity to causal

¡71 confounding, other neuroimaging results are clearly unrelated and, in some cases,

¡72 largely incidental to the specific model implementation. In particular, as can be seen

573 in Figures 6 and 7, the ordinal mean values derived from causal and error-driven

¡74 accounts across trial types during feedback-based learning are identical for both

¡75 strength and surprise measures, suggesting that model-selection may reflect more

j76 granular differences. For example, because of the $\Sigma$ term in Equations 1 and 2 of the

577 error-driven account, the variance in the occurrence of the outcome across e-, De+

j78 and Se \pm trials results in a constantly fluctuating strength, and persistent prediction

579 errors, even on deterministic e- and $\mathrm{De}+$, trials. In contrast, in the implementation of

580 the causal model, such non-conditional variance is eliminated by the use of a focal set

j81 (e.g., Cheng \& Novick, 1992; Spellman, 1996). Behaviorally, the focal set assumption

582 is strongly supported by the lack of variability in judgments about non-target cause e.

583 This cause, which is paired with the outcome on half of trials in which it occurs, but

j84 never when occurring in isolation, is almost exclusively judged to be non-causal, 585 suggesting that participants conditioned their inferences about its influence on trials j86 across which alternative causes could be assumed to be held constant. Nonetheless, ¡87 at the neural level, several regions may instead have been tracking the unconditional 588 outcome variance computed by the error-driven model.

589 As the mantra "covariation does not equal causation" implies, requirements for causal j90 induction extend far beyond mere statistical regularity. Countless studies have ¡91 demonstrated neural and behavioral effects of error-driven uncertainty, whether due to 592 stochasticity, insufficient samples or changes in the statistical structure of the 
593 environment. The current study reveals that, given violation of a basic boundary j94 condition on causal inference, and in spite of the same constraints on perceptual 595 generalization, differences in neural representations of uncertainty can emerge across 596 equally deterministic, static and well-sampled contingencies. These results contribute $j 97$ to a growing literature on the role of generative models in neural representations of 598 uncertainty, and shed light on a possible neural implementation of domain general, a $う 99$ priori, constraints on causal induction. 


\section{Acknowledgements}

503 This work was supported by a start-up fund from the University of California Irvine to 504 M.L. 


\section{References}

509 Abler, B., Herrnberger, B., Grön, G., \& Spitzer, M. (2009). From uncertainty to reward: 510 BOLD characteristics differentiate signaling pathways. BMC neuroscience, 10(1), 511154

512 Allen, M., Fardo, F., Dietz, M. J., Hillebrandt, H., Friston, K. J., Rees, G., \& Roepstorff, 513 A. (2016). Anterior insula coordinates hierarchical processing of tactile mismatch responses. Neuroimage, 127, 34-43.

515 Bach, D. R., Seymour, B., \& Dolan, R. J. (2009). Neural activity associated with the 516 passive prediction of ambiguity and risk for aversive events. Journal of 517 Neuroscience, 29(6), 1648-1656.

518 Baetens, K., Ma, N., Steen, J., \& Van Overwalle, F. (2013). Involvement of the 519 mentalizing network in social and non-social high construal. Social cognitive and 520 affective neuroscience, 9(6), 817-824.

521 Baetens, K. L., Ma, N., \& Van Overwalle, F. (2017). The dorsal medial prefrontal 522 cortex is recruited by high construal of non-social stimuli. Frontiers in behavioral 523 neuroscience, 11, 44.

524 Bastin, J., Deman, P., David, O., Gueguen, M., Benis, D., Minotti, L., ... \& Kahane, P. 525 (2016). Direct recordings from human anterior insula reveal its leading role within 526 the error-monitoring network. Cerebral Cortex, bhv352.

527 Causse, M., Péran, P., Dehais, F., Caravasso, C. F., Zeffiro, T., Sabatini, U., \& Pastor, 528 J. (2013). Affective decision making under uncertainty during a plausible aviation 529 task: An fMRI study. Neurolmage, 71, 19-29. 
Cheng P (1997) From covariation to causation: A causal power theory. Psycholgoical

531 Review:367-405.

532 Cheng, P. W., \& Novick, L. R. (1992). Covariation in natural causal induction.

$533 \quad$ Psychological Review, 99(2), 365.

534 Courville, A. C., Daw, N. D., \& Touretzky, D. S. (2006). Bayesian theories of

535 conditioning in a changing world. Trends in cognitive sciences, 10(7), 294-300.

536 Critchley, H. D., Mathias, C. J., \& Dolan, R. J. (2001). Neural activity in the human

537 brain relating to uncertainty and arousal during anticipation. Neuron, 29(2), 537538545.

539 d'Acremont, M., Fornari, E., \& Bossaerts, P. (2013). Activity in inferior parietal and 540 medial prefrontal cortex signals the accumulation of evidence in a probability 541 learning task. PLoS computational biology, 9(1), e1002895.

542 Durnez, J., Degryse, J., Seurinck, R., Moerkerke, B., \& Nichols, T. E. (2015).

543 Prospective power estimation for peak inference with the toolbox neuropower.

544 In Second Belgian Neuroinformatics Congress (Vol. 9). Frontiers Media SA.

545 Esber, G. R., \& Haselgrove, M. (2011). Reconciling the influence of predictiveness 546 and uncertainty on stimulus salience: a model of attention in associative 547 learning. Proceedings of the Royal Society of London B: Biological $548 \quad$ Sciences, 278(1718), 2553-2561.

549 Egner, T. (2009). Prefrontal cortex and cognitive control: motivating functional 550 hierarchies. Nature neuroscience, 12(7), 821.

551 Fellows, L. K., \& Farah, M. J. (2005). Is anterior cingulate cortex necessary for 552 cognitive control?. Brain, 128(4), 788-796. 
553 Forman, S. D., Cohen, J. D., Fitzgerald, M., Eddy, W. F., Mintun, M. A., \& Noll, D. C.

554 (1995). Improved assessment of significant activation in functional magnetic

555 resonance imaging (fMRI): use of a cluster-size threshold. Magnetic Resonance in 556 medicine, 33(5), 636-647.

557 Glymour C (1998) Psychological and normative theories of causal power and the 558 probabilities of causes. In: Uncertainty in artificial intelligence (F. CG, S. M, eds): 559 Kaufmann.

560 Gläscher, J. (2009) Visualization of group inference data in functional neuroimaging. $561 \quad$ Neuroinformatics, 7(1), 73-82.

562 Griffiths, T. L., \& Tenenbaum, J. B. (2005). Structure and strength in causal 563 induction. Cognitive psychology, 51(4), 334-384.

564 Grinband, J., Hirsch, J., \& Ferrera, V. P. (2006). A neural representation of 565 categorization uncertainty in the human brain. Neuron, 49(5), 757-763.

566 Holt, C. A., \& Laury, S. K. (2002). Risk aversion and incentive effects. American 567 economic review, 92(5), 1644-1655.

568 Hampton, A. N., Bossaerts, P., \& O'doherty, J. P. (2006). The role of the ventromedial 569 prefrontal cortex in abstract state-based inference during decision making in 570 humans. Journal of Neuroscience, 26(32), 8360-8367.

571 Huettel, S. A., Stowe, C. J., Gordon, E. M., Warner, B. T., \& Platt, M. L. (2006). 572 Neural signatures of economic preferences for risk and ambiguity. Neuron, 49(5), $573 \quad 765-775$. 
574 Huettel SA, Song AW, McCarthy G. (2005) Decisions under uncertainty: probabilistic $575 \quad$ context influences activity of prefrontal and parietal cortices. J Neurosci. 25:3304$576 \quad 3311$

577 Hsu, M., Bhatt, M., Adolphs, R., Tranel, D., \& Camerer, C. F. (2005). Neural systems 578 responding to degrees of uncertainty in human decision579 making. Science, 310(5754), 1680-1683.

580 Itti, Laurent, and Pierre F. Baldi. "Bayesian surprise attracts human 581 attention." Advances in neural information processing systems. 2006.

582 Kerns, J. G., Cohen, J. D., MacDonald, A. W., Cho, R. Y., Stenger, V. A., \& Carter, C. 583 S. (2004). Anterior cingulate conflict monitoring and adjustments in $584 \quad$ control. Science, 303(5660), 1023-1026.

585 Klein, T. A., Endrass, T., Kathmann, N., Neumann, J., von Cramon, D. Y., \& 586 Ullsperger, M. (2007). Neural correlates of error awareness. Neuroimage, 34(4), $587 \quad$ 1774-1781.

588 Krain, A. L., Wilson, A. M., Arbuckle, R., Castellanos, F. X., \& Milham, M. P. (2006). 589 Distinct neural mechanisms of risk and ambiguity: a meta-analysis of decision590 making. Neuroimage, 32(1), 477-484.

591 Kushnir T, Gopnik A (2005) Young children infer causal strength from probabilities and $592 \quad$ interventions. Psychological science 16:678-683.

593 Levy, I., Snell, J., Nelson, A. J., Rustichini, A., \& Glimcher, P. W. (2010). Neural 594 representation of subjective value under risk and ambiguity. Journal of 595 neurophysiology, 103(2), 1036-1047. 
596 Liljeholm, M., \& Cheng, P. W. (2009). The influence of virtual sample size on

597 confidence and causal-strength judgments. Journal of Experimental Psychology:

598 Learning, Memory, and Cognition, 35(1), 157.

599 Liljeholm, M. (2015). How multiple causes combine: independence constraints on $700 \quad$ causal inference. Frontiers in psychology, 6.

701 Lu, H., Yuille, A. L., Liljeholm, M., Cheng, P. W., \& Holyoak, K. J. (2008). Bayesian 702 generic priors for causal learning. Psychological review, 115(4), 955.

703 Botvinick, M. M., Braver, T. S., Barch, D. M., Carter, C. S., \& Cohen, J. D. (2001).

704 Conflict monitoring and cognitive control. Psychological review, 108(3), 624.

705 Meder B, Hagmayer Y, Waldmann M (2006) Understanding the Causal Logic of 706 Confounds. In: Twenty-Eighth Annual Conference of the Cognitive Science $707 \quad$ Society, pp 579-584: Erlbaum.

708 Metereau, E., \& Dreher, J. C. (2012). Cerebral correlates of salient prediction error for 709 different rewards and punishments. Cerebral Cortex, 23(2), 477-487.

710 Mohr, P. N., Biele, G., \& Heekeren, H. R. (2010). Neural processing of risk. Journal of $711 \quad$ Neuroscience, 30(19), 6613-6619.

712 Nichols, T. E., \& Holmes, A. P. (2002). Nonparametric permutation tests for functional 713 neuroimaging: a primer with examples. Human brain mapping, 15(1), 1-25.

714 Payzan-LeNestour, E., Dunne, S., Bossaerts, P., \& O’Doherty, J. P. (2013). The 715 neural representation of unexpected uncertainty during value-based decision 716 making. Neuron, 79(1), 191-201. 
717 Payzan-LeNestour, E., \& Bossaerts, P. (2011). Risk, unexpected uncertainty, and

718 estimation uncertainty: Bayesian learning in unstable settings. PLoS computational

719 biology, 7(1), e1001048.

720 Pearce, J. M. (1987). A model for stimulus generalization in Pavlovian

721 conditioning. Psychological review, 94(1), 61.

722 Pearce, J. M. (2002). Evaluation and development of a connectionist theory of

723 configural learning. Animal Learning \& Behavior, 30(2), 73-95.

724 Preuschoff, K., Quartz, S. R., \& Bossaerts, P. (2008). Human insula activation reflects

725 risk prediction errors as well as risk. Journal of Neuroscience, 28(11), 2745-2752.

726 Prévost, C., McNamee, D., Jessup, R. K., Bossaerts, P., \& O'Doherty, J. P. (2013).

727 Evidence for model-based computations in the human amygdala during Pavlovian

728 conditioning. PLoS computational biology, 9(2), e1002918.

729 Pushkarskaya, H., Smithson, M., Joseph, J. E., Corbly, C., \& Levy, I. (2015). Neural 730 correlates of decision-making under ambiguity and conflict. Frontiers in behavioral 731 neuroscience, 9, 325.

732 Richiardi, J., Altmann, A., Milazzo, A. C., Chang, C., Chakravarty, M. M., 733 Banaschewski, T., ... \& Conrod, P. (2015). Correlated gene expression supports 734 synchronous activity in brain networks. Science, 348(6240), 1241-1244.

735 Ridderinkhof, K. R., Ullsperger, M., Crone, E. A., \& Nieuwenhuis, S. (2004). The role 736 of the medial frontal cortex in cognitive control. science, 306(5695), 443-447.

737 Rosa, M. J., Bestmann, S., Harrison, L., \& Penny, W. (2010). Bayesian model 738 selection maps for group studies. Neuroimage, 49(1), 217-224. 
739 Schulz L, Bonawitz E (2007) Serious fun: preschoolers engage in more exploratory 740 play when evidence is confounded. Developmental psychology 43:1045-1050.

741 Schulz L, Gopnik A, Glymour C (2007) Preschool children learn about causal structure 742 from conditional interventions. Dev Sci 10:322-332.

743 Shenhav, A., Botvinick, M. M., \& Cohen, J. D. (2013). The expected value of control:

744 an integrative theory of anterior cingulate cortex function. Neuron, 79(2), 217-240.

745 Spellman B (1996) Acting as Intuitive Scientists: Contingency Judgments Are Made

746 While Controlling for Alternative Potential Causes. Psychological science 7:337$747 \quad 342$.

748 Taren, A. A., Venkatraman, V., \& Huettel, S. A. (2011). A parallel functional

749 topography between medial and lateral prefrontal cortex: evidence and

750 implications for cognitive control. Journal of Neuroscience, 31(13), 5026-5031.

751 Volz KG, Schubotz RI, von Cramon DY. (2003) Predicting events of varying

752 probability: uncertainty investigated by fMRI. Neuroimage. ;19:271-280.

753 Xue, G., Lu, Z., Levin, I. P., Weller, J. A., Li, X., \& Bechara, A. (2008). Functional

754 dissociations of risk and reward processing in the medial prefrontal

$755 \quad$ cortex. Cerebral Cortex, 19(5), 1019-1027.

756

757

758

759 
761 Figure 1. Task illustration. A. Prediction and outcome screens from a feedback-

762 based learning trial. Screens were separated by a $2 \mathrm{sec}$. pause during which the 763 word "Wait" (not shown in figure) was displayed.

B. Causal query regarding the

764 individual influence of a particular medicine on headache.

765 Figure 2. Possible causal structures involving a background cause, B, a target cause,

766 C, an alternative cause, A, and an effect, E. A link to the effect always exists for B, 767 and may exist for neither $\mathrm{C}$ nor $\mathrm{A}\left(\mathrm{Graph}_{0}\right)$, C only $\left(\mathrm{Graph}_{1}\right)$, or both $\mathrm{C}$ and $\mathrm{A}\left(\mathrm{Graph}_{2}\right)$.

768 Figure 3. Mean (normalized) probabilities of uncertain "May or may not be a cause"

769 judgments derived from the Bayesian causal model and error-driven algorithm

770 respectively. Confounded, deterministic and stochastic target causes (C, D \& S)

771 occurred only in compound with alternative causes during feedback-based learning,

772 while non-target causes occurred only individually (s'), or individually as well as in

773 compound (e). Error bars=SEM.

774 Figure 4. Selective effects of the causal model of uncertainty during judgments about

775 the causal influences of individual medicines, showing effects in the dorsomedial and 776 dorsolateral prefrontal cortex (DMPFC and DLPFC), and the lateral Superior Frontal 777 Gyrus (LSFG). Bar plots show effect sizes extracted from spheres centered on peak 778 coordinates for each queried medicine. Error bars=SEM.

779 Figure 5. Selective effects of the error-driven model of uncertainty during the 780 prediction period of feedback-based learning trials, showing effects in the right anterior 
781 insula (aINS), posterior middle temporal gyrus (pMTG) and lingual gyrus (LG). Bar

782 plots show effect sizes extracted from spheres centered on peak coordinates for each

783 type of medicine trial. Error bars=SEM.

784 Figure 6. Strength predictions derived from the causal and error-driven model (top),

785 together with effect sizes (bottom) extracted from spheres centered on peak

786 coordinates in the precentral gyrus, for each type of medicine trial during feedback-

787 based learning. Error bars=SEM.

788 Figure 7. Outcome surprise signals derived from the causal and error-driven model

789 (top), together with effect sizes (bottom) extracted from spheres centered on peak

790 coordinates in the anterior caudate nucleus (aCN) and anterior insula (aINS), for each

791 type of medicine trial during feedback-based learning. Error bars=SEM. 
Table 1. Mean proportions of "Is a cause", "Is not a cause" and "May or may

796

797 not be a cause" judgments, and associated response times, with standard deviations, for target causes, C, D and S, and non-target causes, s' and e'.

\begin{tabular}{c|c|c|c|c|c}
\hline & C & D & S & $s^{\prime}$ & e \\
\hline Proportions & & & & & \\
Is a cause & $0.36 \pm 0.33$ & $0.84 \pm 0.25$ & $0.38 \pm 0.25$ & $0.40 \pm 0.27$ & $0.02 \pm 0.03$ \\
Is not a cause & $0.03 \pm 0.04$ & $0.06 \pm 0.09$ & $0.23 \pm 0.11$ & $0.30 \pm 0.17$ & $0.96 \pm 0.05$ \\
May be a cause & $0.61 \pm 0.35$ & $0.10 \pm 0.21$ & $0.39 \pm 0.31$ & $0.30 \pm 0.28$ & $0.02 \pm 0.04$ \\
Response Times & & & & & \\
Is a cause & $2.92 \pm 1.88$ & $2.52 \pm 1.23$ & $4.03 \pm 3.62$ & $2.82 \pm 2.59$ & $2.86 \pm 2.11$ \\
Is not a cause & $4.26 \pm 2.69$ & $3.94 \pm 3.36$ & $4.19 \pm 3.64$ & $2.72 \pm 1.23$ & $2.44 \pm 1.15$ \\
May be a cause & $3.79 \pm 2.68$ & $3.76 \pm 1.46$ & $2.99 \pm 1.94$ & $2.59 \pm 1.32$ & $4.08 \pm 4.78$
\end{tabular}


Table 2: Non-masked significant, whole-brain corrected, neural effects of the causal model of uncertainty, strength and surprise, during causal judgments and feedback-based learning, with peak MNI coordinates and cluster sizes at $p<0.001$. $\mathrm{R} / \mathrm{L}=$ Right/Left, $(-)=$ negative correlation .

\begin{tabular}{|c|c|c|c|}
\hline Contrast & Region & Peak MNI & Cluster Size \\
\hline \multirow[t]{3}{*}{ Judgment Uncertainty } & DMPFC & $-8,28,46$ & 1900 \\
\hline & R. IPG & $50,-52,40$ & 292 \\
\hline & Dorsolateral PFC & $42,34,30$ & 201 \\
\hline \multirow[t]{5}{*}{ Prediction Uncertainty } & Fusiform & $28,-72,-12$ & 7195 \\
\hline & DMPFC & $-10,16,52$ & 1387 \\
\hline & R. Insula & $-30,18,4$ & 726 \\
\hline & L. Insula & $34,30,-4$ & 470 \\
\hline & Thalamus & $6,-22,2$ & 867 \\
\hline Prediction Strength & Calcarine/Occipital & $18,-96,4$ & 236 \\
\hline \multirow[t]{5}{*}{ Prediction Strength (-) } & Lingual Gyrus & $14,-62,-2$ & 2232 \\
\hline & Superior Frontal Gyrus & $-26,-8,58$ & 458 \\
\hline & Superior Parietal Lobule & $-28,-50,62$ & 304 \\
\hline & Inferior Parietal Gyrus & $-46,-26,36$ & 336 \\
\hline & Supp. Motor Area & $8,2,52$ & 297 \\
\hline \multirow[t]{5}{*}{ Surprise } & Right Insula & $36,18,-2$ & 652 \\
\hline & Left Insula & $-28,22,-6$ & 482 \\
\hline & Dorsomedial PFC & $4,46,34$ & 1146 \\
\hline & Left Dorsolateral PFC & $-46,4,42$ & 192 \\
\hline & Right Dorsolateral PFC & $44,24,30$ & 325 \\
\hline \multirow[t]{6}{*}{ Surprise (-) } & Supramarginal Gyrus & $-50,-26,38$ & 1858 \\
\hline & Caudate & $18,32,0$ & 505 \\
\hline & Mid Cingulum & $-10,-30,42$ & 472 \\
\hline & Postcentral Gyrus & $50,-20,48$ & 2399 \\
\hline & Parahippocampal & $34,-32,-12$ & 227 \\
\hline & Precuneus & $16,-52,14$ & 221 \\
\hline
\end{tabular}


Table 3: Non-masked significant, whole-brain corrected, neural effects of the error-driven model of uncertainty, strength and surprise during feedback-based learning, with peak $\mathrm{MNI}$ coordinates and cluster sizes at $\mathrm{p}<0.001$. $\mathrm{R} / \mathrm{L}=$ Right/Left, $(-)=$ negative correlation .

\begin{tabular}{|c|c|c|c|}
\hline Contrast & Region & Peak MNI & Cluster Size \\
\hline \multirow[t]{4}{*}{ Prediction Uncertainty } & Calcarine/Lingual Gyrus & $14,-88,0$ & 3116 \\
\hline & Supp. Motor Area & $10,20,48$ & 153 \\
\hline & Left Insula & $-24,22,2$ & 65 \\
\hline & Right Insula & $34,28,-2$ & 109 \\
\hline \multirow[t]{4}{*}{ Prediction Uncertainty (-) } & Middle Temporal Gyrus & $-46,-62,20$ & 341 \\
\hline & Middle Temporal Gyrus & $-56,-22,-10$ & 84 \\
\hline & Ventromedial PFC & $-2,30,-14$ & 157 \\
\hline & Middle Temporal Gyrus & $46,-58,22$ & 64 \\
\hline Prediction Strength & Lingual Gyrus & $-20,-78,-10$ & 2801 \\
\hline \multirow[t]{7}{*}{ Prediction Strength (-) } & Superior Parietal Lobule & $-18,-52,64$ & 407 \\
\hline & Supramarginal Gyrus & $-60,-28,30$ & 390 \\
\hline & Precentral Gyrus & $26,-12,50$ & 240 \\
\hline & Supramarginal Gyrus & $58,-38,28$ & 728 \\
\hline & Middle Temporal Gyrus & $-54,-60,2$ & 603 \\
\hline & Precentral Gyrus & $-22,-14,64$ & 383 \\
\hline & Thalamus & $-12,-10,-4$ & 214 \\
\hline \multirow[t]{6}{*}{ Prediction Error } & Right Insula & $38,18,-4$ & 912 \\
\hline & Left Insula & $-40,20,4$ & 945 \\
\hline & Precentral Gyrus & $-42,-2,40$ & 713 \\
\hline & Anterior Cingulate & $4,38,30$ & 2021 \\
\hline & Inferior Parietal Gyrus & $-28,-52,42$ & 246 \\
\hline & Dorsolateral PFC & $44,28,26$ & 885 \\
\hline \multirow[t]{3}{*}{ Prediction Error (-) } & Middle Occipital Gyrus & $-42,-74,26$ & 735 \\
\hline & Fusiform Gyrus & $-34,-42,-12$ & 253 \\
\hline & Postcentral Gyrus & $54,-26,52$ & 906 \\
\hline
\end{tabular}

